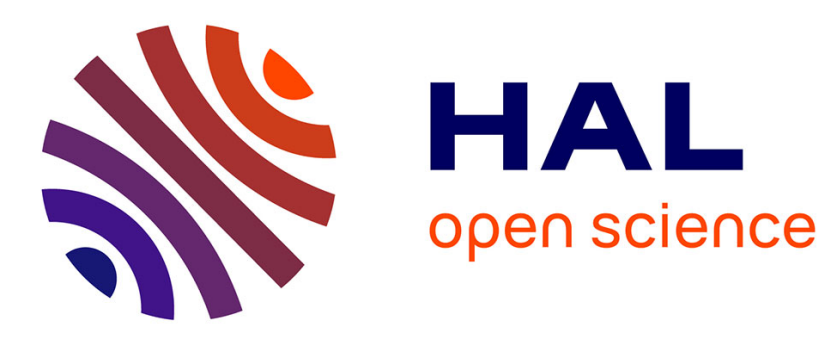

\title{
Design Method for Chemical Clogging Emitters Boundary Optimization
}

Xu Li, Peiling Yang, Shumei Ren, Lili Zhangzhong, Lihong Yang

\section{To cite this version:}

Xu Li, Peiling Yang, Shumei Ren, Lili Zhangzhong, Lihong Yang. Design Method for Chemical Clogging Emitters Boundary Optimization. 10th International Conference on Computer and Computing Technologies in Agriculture (CCTA), Oct 2016, Dongying, China. pp.318-329, 10.1007/978-3-03006155-5_32.hal-02179983

\section{HAL Id: hal-02179983 https://hal.inria.fr/hal-02179983}

Submitted on 12 Jul 2019

HAL is a multi-disciplinary open access archive for the deposit and dissemination of scientific research documents, whether they are published or not. The documents may come from teaching and research institutions in France or abroad, or from public or private research centers.
L'archive ouverte pluridisciplinaire HAL, est destinée au dépôt et à la diffusion de documents scientifiques de niveau recherche, publiés ou non, émanant des établissements d'enseignement et de recherche français ou étrangers, des laboratoires publics ou privés.

\section{(c)(1)}

Distributed under a Creative Commons Attribution| 4.0 International License 


\title{
Design Method for Chemical Clogging Emitters Boundary Optimization
}

\author{
Xu Li ${ }^{1, \text { a }}$, Peiling Yang ${ }^{1, b(\bowtie)}$, Shumei Ren ${ }^{1, \mathrm{c}}$,Lili Zhangzhong ${ }^{2, \mathrm{~d}}$, Lihong Yang ${ }^{3, \mathrm{e}}$, \\ 1 College of Water Resources \& Civil Engineering, China Agricultural University, Beijing 100083,China; \\ 2 China National Engineering Research Center for Information Technology in Agriculture,Beijing,100097,China \\ 3 Yunnan Academy of Scientific\&Technical Information,Yunnan, 650051,China; \\ afengyunlixu@163.com, ${ }^{b}$ yangpeiling@126.com, ${ }^{\mathrm{c}}$ renshumei@126.com, ${ }^{\mathrm{d}}$ zhangzll@nercita.org.cn, \\ eylhlee8956@126.com
}

\begin{abstract}
Fractal flow channel structure as research object, based on chemical clogging condition of the physical model. It was analyzed using computational fluid dynamics (CFD) simulation and reveals the fractal flow channel internal flow characteristics of water and sediment. Fractal flow channel non-energy dissipation of the arc angle design optimization. Using standard $\kappa-\varepsilon$ turbulence model and the DPM model, calculated: (1) As for the hydraulic performance analysis, before optimization emitter flow exponent of 0.487, 0.489 after optimization; From the inner flow field analysis, When the pressure head from $5 \mathrm{~m}$ to $15 \mathrm{~m}$, before optimization emitter maximum flow rate from $2.09 \mathrm{~m} / \mathrm{s}$ to $3.70 \mathrm{~m} / \mathrm{s}$, the maximum flow rate to optimize the emitter from $2.15 \mathrm{~m} / \mathrm{s}$ to $3.81 \mathrm{~m} / \mathrm{s}$, the maximum optimization of flow rates were increased compared to the previous $2.87 \%$, $3.34 \%, 2.97 \%$,the flow rate improved. After optimizing the eddy region, the velocity of the outer edge of the eddy region increased from $(0.005-0.752 \mathrm{~m} / \mathrm{s})$ to $(0.311-0.930 \mathrm{~m} / \mathrm{s})$, which improved the self-cleaning ability of the irrigator. Based on the analysis of blockage performance, the passing rate of particles is significantly improved after optimizing the flow channel. Considering the optimized emitter has excellent hydraulic performance and anti-clogging properties.
\end{abstract}

Keyword: Fractal runner · Hydraulic performance · Anti-clogging properties · Numerical simulation · Structural optimization

\section{Introduction}

Drip irrigation as an effective water-saving irrigation technology has irrigation uniformity, water and fertilizer, yield, ease of management, etc., in order to achieve the refinement of farming and agriculture yield, high quality, efficient and provide the conditions, so by the widespread attention at home and abroad[1-3]. Irrigation is a core component of the entire irrigation system [4、5], accounting for $15 \%$ to $20 \%$ [6] of the total system cost. However, the emitter blockage has been long plagued the development of drip irrigation technology, which directly affect the promotion and use of the effect of drip irrigation technology. Structure and hydraulic performance have strong impact on the drip 
irrigation system, irrigation uniformity, a large anti-clogging performance, life span of the system [7、 8]. Therefore it is pretty necessary to expand research about the impact of the flow channel structure within the emitter flow channel flow regime, hydraulic performance and anti-clogging properties. At present, domestic and foreign scholars have applied the CFD numerical simulation method to optimize the design of runner emitter. Wei et al [9、10]. By rectangles and zigzag flow channel conduct computational fluid dynamics analysis and two-phase flow analysis, he pointed out that the low-speed corners at the vortex zone is caused by blockage of the focus position, to ensure the flow channel hydraulic performance on the basis of the main channel anti-clogging design optimization. Li et al. [11] has chosen the Chinese agricultural irrigation areas most typical three cylindrical emitter simulation analysis, to achieve the internal flow channel velocity distribution, vorticity distribution, flow characteristics of turbulence intensity visualization, providing a new theory developed irrigation flow passage basis. Wang et al.[12] The key structure parameters based on a triangular circulation flow path for the factors, the influence of structural parameters on the hydraulic performance of the emitter flow coefficient and flow exponent for the test index orthogonal numerical simulation test. Yan et al. [13] used CFD simulation technology, different concentrations within the labyrinth flow channel emitter suspended particles movement digitized simulation, using the kinetic theory of two-phase flow with suspended particles popular labyrinth emitter structure scales were analyzed study found that the use DPM model for distribution of suspended particles in the simulation results with the experimental data fit a higher degree. But overall, the current research is mainly related to flow channel from the emitter structure unit parameter changes the perspective of hydraulic performance and the performance of anti-clogging flow passage, not based within the chemical conditions clogged emitters flow channel Flow Pattern Analysis and the movement of particulate matter research, ignoring the movement of sediment has been blocked in case of a chemical.

Based on this, select Li [14] designed and developed Minkowski fractal flow channel emitter, and select an optimal flow channel dimensions flow channel boundary optimization design based on existing research. Standard $\kappa-\varepsilon$ model and the DPM model numerical calculation, the hydraulic performance of emitters (flow exponent), the flow field distribution and anti-clogging ability (particles passing rate) were compared. The results can provide reference for the research and design of emitter.

\section{Experiments and Methods}

\subsection{Model establishment}

\subsubsection{The standard $k-\varepsilon$ model}

Continuity equation

Continuity equations:

$$
\frac{\partial u}{\partial x}+\frac{\partial v}{\partial y}+\frac{\partial w}{\partial z}=0
$$

Momentum equations:

$$
\begin{gathered}
\frac{\partial(\rho u)}{\partial t}+\nabla \cdot(\rho u \boldsymbol{u})=\mu \nabla^{2} u-\frac{\partial p}{\partial x}+S_{u} \\
\frac{\partial(\rho v)}{\partial t}+\nabla \cdot(\rho v \boldsymbol{u})=\mu \nabla^{2} v-\frac{\partial p}{\partial y}+S_{v}
\end{gathered}
$$




$$
\frac{\partial(\rho w)}{\partial t}+\nabla \cdot(\rho w \boldsymbol{u})=\mu \nabla^{2} w-\frac{\partial p}{\partial z}+S_{w}
$$

Standard k- $\varepsilon$ equations:

$$
\begin{gathered}
\frac{\partial(\rho k)}{\partial t}+\frac{\partial\left(\rho k u_{i}\right)}{\partial x_{i}}=\frac{\partial}{\partial x_{j}}\left[\left(\mu+\frac{\mu_{t}}{\sigma_{k}}\right) \frac{\partial k}{\partial x_{j}}\right]+G_{k}-\rho \varepsilon \\
\frac{\partial(\rho \varepsilon)}{\partial t}+\frac{\partial\left(\rho \varepsilon u_{i}\right)}{\partial x_{i}}=\frac{\partial}{\partial x_{j}}\left[\left(\mu+\frac{\mu_{t}}{\sigma_{\varepsilon}}\right) \frac{\partial \varepsilon}{\partial x_{j}}\right]+\frac{C_{1 \varepsilon}}{k} G_{k}-C_{2 \varepsilon} \rho \frac{\varepsilon^{2}}{k}
\end{gathered}
$$

In the equations above, $\mathbf{u}$ represents the velocity vector $; \mathrm{u} 、 \mathrm{v}$ and $\mathrm{w}$ directions velocity component ; $\mathrm{Su} 、 \mathrm{~Sv}$ and $\mathrm{Sw}$ are the generalized source term ; $\mathbf{u}_{\mathrm{i}}$ stands for mean velocity; $\mu_{\mathrm{t}}$ stands for turbulent viscosity ; $\mathrm{k}$ stands for turbulent kinetic energy ; $\varepsilon$ stands for dissipation rate $; \mathrm{G}_{\mathrm{k}}$ stands for the average velocity gradient caused by the turbulent kinetic energy term. The values of coefficients are $\mathrm{C}_{1 \varepsilon}=1.44 ; \mathrm{C}_{2 \varepsilon}=1.92 ; \sigma_{\mathrm{k}}=1.0 ; \sigma_{\varepsilon}=1.3$ respectively.

\subsubsection{The DPM model}

In calculating the equations of motion particles, the particles in the flow channel are mainly affected by the drag force, gravity. And other additional force such as pressure gradient force, false mass force, Saffman lift, thermal swimming Basset force are smaller than the former, so we don't care.

The equations of motion of a single particle derived directly from Newton's second law:

$$
m_{p} \frac{d u_{p}}{d t}=F_{d}+F_{M}
$$

Where $\mathrm{m}_{\mathrm{p}}$ is defined as $m_{p}=\pi \rho_{p} d_{p}^{3} / 6$, it stands for mass particles. $u_{p}(\mathrm{~m} / \mathrm{s})$ represents particle velocity; $\mathrm{F}_{\mathrm{D}}$ stands for fluid drag force on the particles:

$$
\begin{gathered}
F_{D}=\frac{\pi \mu d_{p}}{8} C_{D} R e_{p}\left(u_{f}-u_{p}\right) \\
C_{D}=a_{1}+\frac{a_{2}}{R e_{p}}+\frac{a_{3}}{R e_{p}^{2}} \\
R e_{p}=\frac{\rho_{d} d_{p}\left|u_{p}-u_{f}\right|}{\mu}
\end{gathered}
$$

In the equations above, $u_{f}$ stands for fluid phase velocity (The average velocity and turbulence and velocity of the fluid); $\mu(\mathrm{N} \cdot \mathrm{s} / \mathrm{m} 2)$ stands for fluid dynamic viscosity; $\rho_{p}(\mathrm{~kg} / \mathrm{m} 3)$ particle density; $d_{p}$ (m) particle diameter; $R e_{p}$ particles Reynolds; $C_{D}$ drag coefficient; a1, a2 and a3 are the constant; FM is defined as $F_{M}=\frac{\pi\left(\rho_{p}-\rho\right) g d_{p}^{3}}{6}$.

Table 1. Geometry parameters of the emitter

\begin{tabular}{ccccc}
\hline Emitter width $(\mathrm{mm})$ & Emitter length $(\mathrm{mm})$ & Path depth $(\mathrm{mm})$ & Path width(mm) & 0.9 \\
\hline 7 & 30 & 1 \\
\hline
\end{tabular}

(a) The emitter 


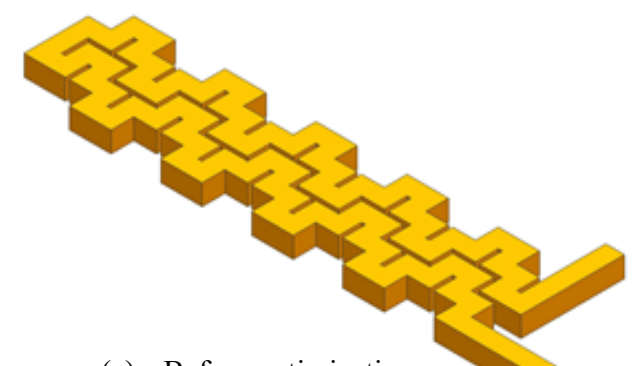

(c) Before optimization

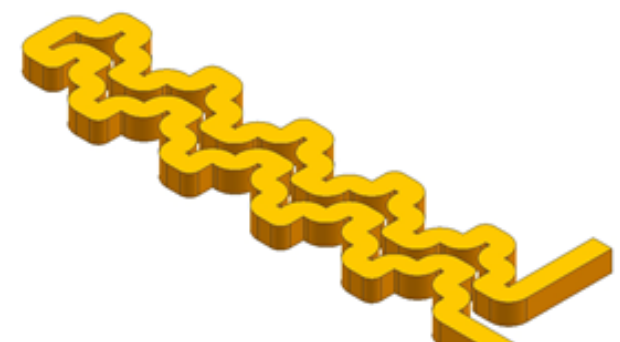

(b) After optimization

Fig. 1. The structure of the irrigation emitter

\section{2 design basis and optimization ideas}

\subsubsection{Design basis}

Currently emitter flow channel type used mainly: labyrinth flow channel, orifice, vortex, micro-irrigation flow channel type, etc. [15、16]. Where in the labyrinth flow path is considered to be the best runner. Through his complex flow path boundary is where the water has reached the purpose of energy dissipation disorder, wherein the main form of energy dissipation for the local pressure loss [14]. However, due to the labyrinth flow path boundary is usually make use of Euclidean geometry principles generated a very limited degree of complexity. Thus the water flow within the flow path can not achieve full turbulence [17]. The fractal flow channel is performed based on fractal theory flow channel design with a high degree of complexity of the flow channel, increasing the energy dissipation capacity of flow path.

\subsubsection{Optimization ideas}

Flow channel designed according to fractal theory is shown in fig. 1 (a). The fractal channel element is more complex than the labyrinth channel unit, and the energy dissipation is greater. Because the fractal Flow channel has a good similarity, flow turning angle is much smaller compared to the labyrinth path, so the water has a good adherent properties, able to play the emitter side walls scouring effect.

Non-circular Flow channel to optimize the structure of energy dissipation turning point in the flow channel width length of the radius of the emitter of the Flow channel for circular, with streamlined design ideas in order to improve anti-clogging ability emitter. Before and after optimization of three-dimensional modeling and optimization emitter flow channel is shown in fig. 1(b), (c).

\subsection{Boundary conditions}

Boundary conditions: the velocity was set as zero at boundaries except the inlet and outlet of the flow path. Channel inlet was set as inlet pressure. Outflow was set as atmospheric pressure.

Initial conditions: inlet working at a certain pressure head, outlet with atmospheric pressure. As the size of the flow channel was small, boundary layer effects could not be ignored, so the standard wall function was employed to solve the problem. 


\section{Results and Discussion}

\subsection{Hydraulic performance}

Fig. 2 showed the hydraulic characteristic curves, Simulate different working pressure conditions obtained before optimization through CFD emitter rated flow 3.62L/h, optimized emitter rated flow 3.67L/h. Use SPSS software to fit the results before optimization emitter flow regime index of 0.487 , emitter optimized flow pattern index of 0.489 , due to the state when the emitter flow index of less than 0.5 is considered an internal turbulent flow regime, with good effect of energy dissipation. Calculated before the optimization and optimized flow exponent emitter little change. After the description of the emitters optimize hydraulic performance of emitters has not been greatly affected.

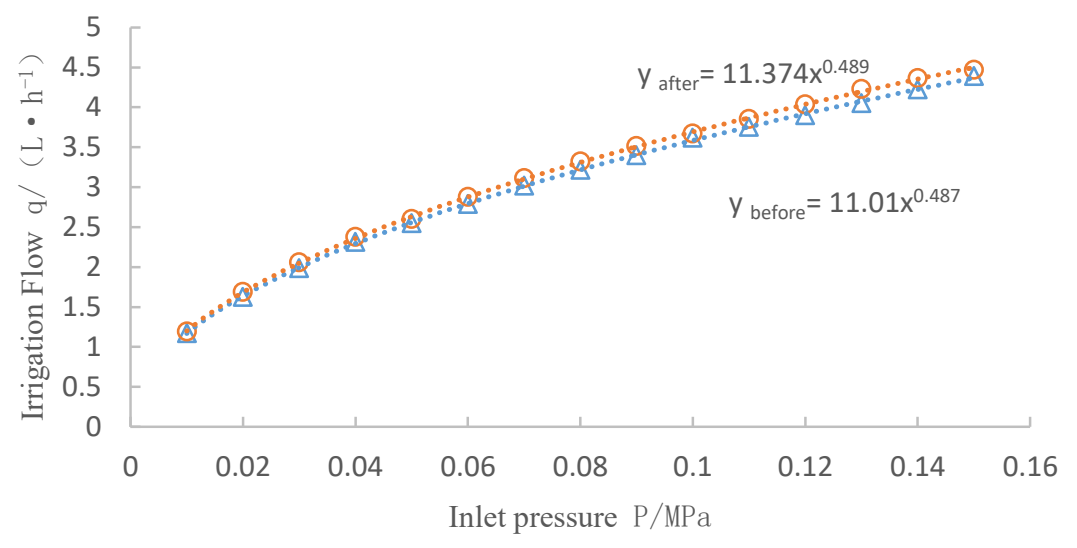

Fig. 2. Hydraulic characteristic curve

\subsection{Internal flow field}

Flow field based on CFD simulation visualization of internal emitters microscopic distribution, Fig. 3 respectively before and after optimization of irrigation's velocity vector in $5 \mathrm{~m}, 10 \mathrm{~m}$ and $15 \mathrm{~m}$ pressure head conditions. As can be seen from the figure, the central portion of the flow channel to form a clear, high-speed mainstream area, near the same location in the flow channel wall tooth angle region are low velocity vortex region. Twists and turns along the flow path, the main area and the particle velocity vortex region continue to occur blended throughout the flow channel exhibits a complicated turbulent state. Before optimization emitters in $5 \mathrm{~m}, 10 \mathrm{~m}$ and $15 \mathrm{~m}$ pressure head maximum flow rate was $2.09 \mathrm{~m} / \mathrm{s}, 2.99 \mathrm{~m} / \mathrm{s}$ and $3.70 \mathrm{~m} / \mathrm{s}$; optimized irrigation in $5 \mathrm{~m}, 10 \mathrm{~m}$ and $15 \mathrm{~m}$ pressure head maximum flow rate was $2.15 \mathrm{~m} / \mathrm{s}, 3.09 \mathrm{~m} / \mathrm{s}$ and $3.81 \mathrm{~m} / \mathrm{s}$. After optimization under the same pressure conditions, Emitters' maximum flow rate were increased $2.87 \%$, 3.34\%, 2.97\% compared to the previous optimization. As the pressure increases, the flow velocity of the flow channel area of the mainstream has improved significantly. Changes in flow velocity and flow characteristics in size did not change significantly after the main area before and after optimization. 


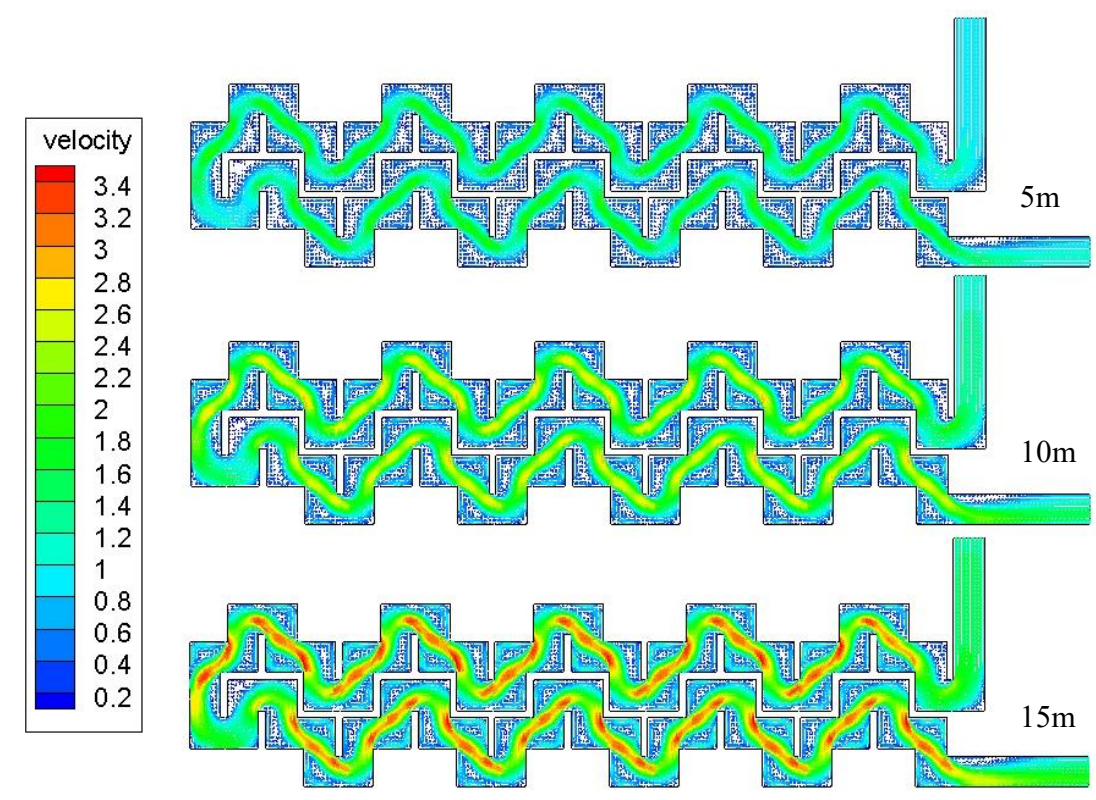

(a) Before optimization emitter velocity vector profile

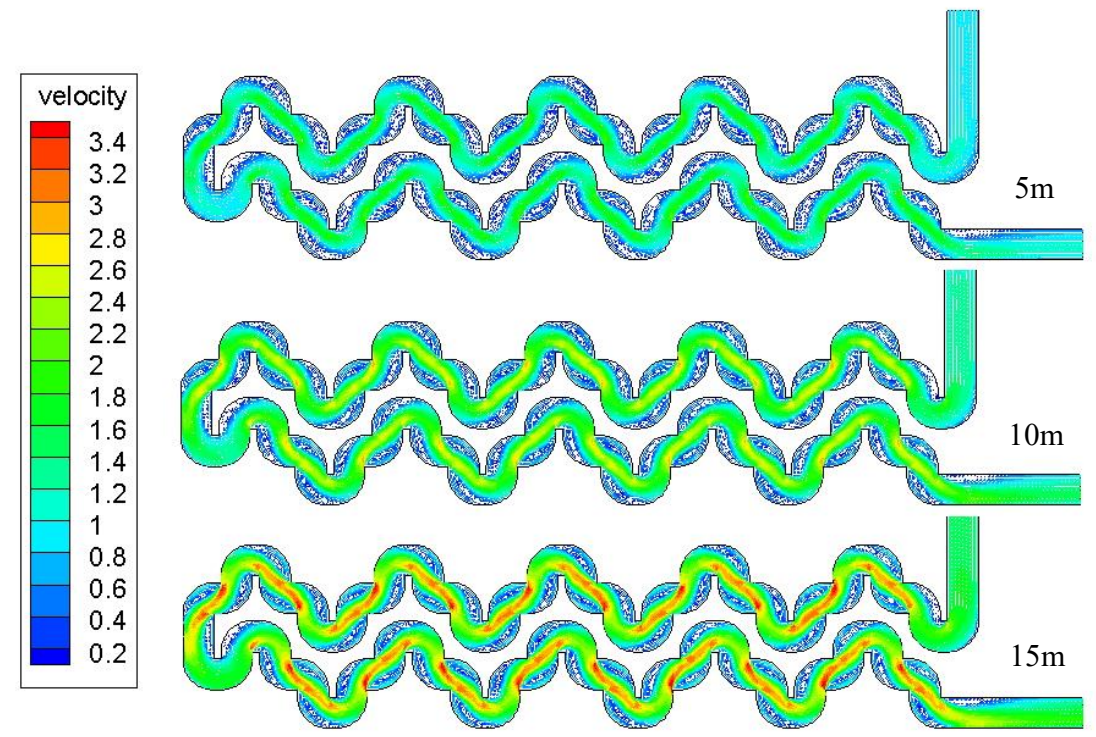

(b) Before optimization emitter velocity vector profile

Fig. 3. Emitter flow velocity vector distribution

Before optimization and optimized flow emitters local vector analysis (in 10m pressure head), we can see that after the optimization of the emitter area than the water back corner vortex before optimization emitters corner carry water vortex area significantly reduced. Outer perimeter of the vortex speeds are $(0.005-0.752 \mathrm{~m} / \mathrm{s})$ and inner $(0.311-0.930 \mathrm{~m} / \mathrm{s})$. From the pictures we can see that the outer edge of the scroll area was significantly higher than the flow rate of the emitter unoptimized emitters. Generally it considered appropriate to increase the emitter area near the wall of the vortex flow rates can be achieved emitter self-cleaning ability. From this perspective, the optimized emitter has a good self-cleaning ability to improve the anti-blocking performance emitter. 


\subsection{Anti-clogging performance}

\subsubsection{DPM Particle Tracking}

During the test, the particles to import jet plane way to have channel inlet plane into the flow path, the initial velocity of the particles and the water flow at the same rate as imports. CFD simulations by particles with different levels of computing, Figure 4 is a particle trajectories track 9, the color indicates the speed of the particle size. Form the results calculated by the trajectory analysis, the particle has the same speed with the flow velocity The particle velocity in the central of flow channel is consistent to the flow velocity, the speed decreases near the wall and vortex area. Fig. 10 showed the particle trajectories of the vast majority particles is basically the same to de main stream trajectory, it has a good following property, so it can flow out with the mainstream; But some particle trajectories showed a different degree
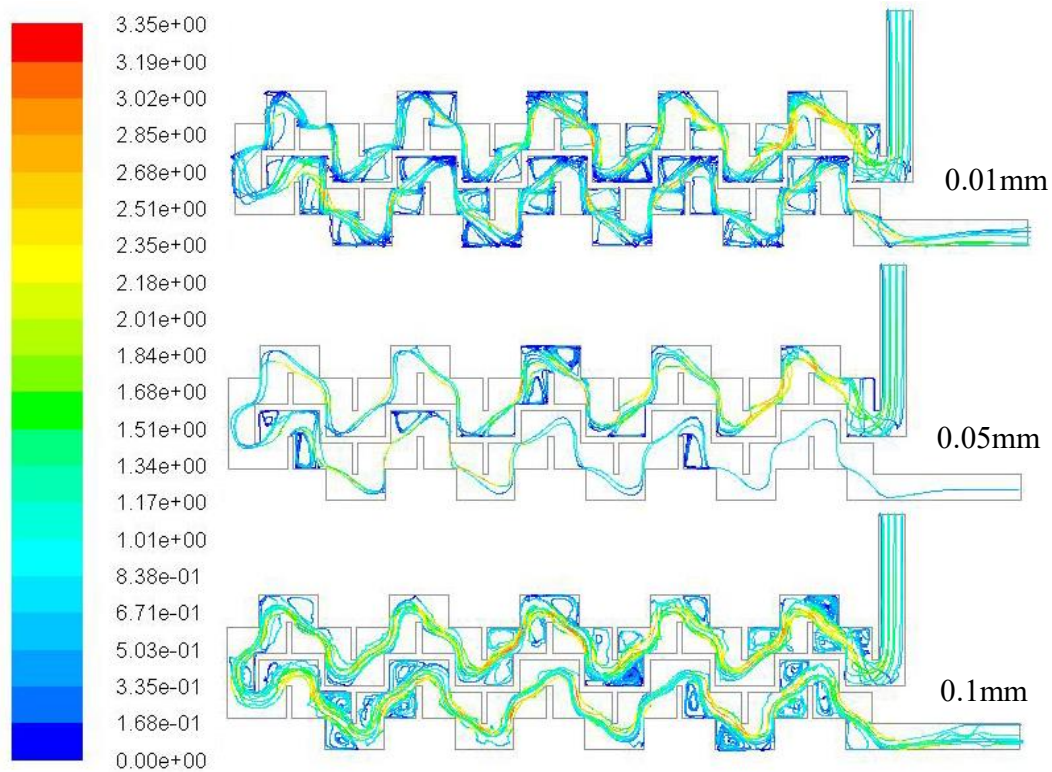

(a) Before optimization within emitter nine particle trajectories

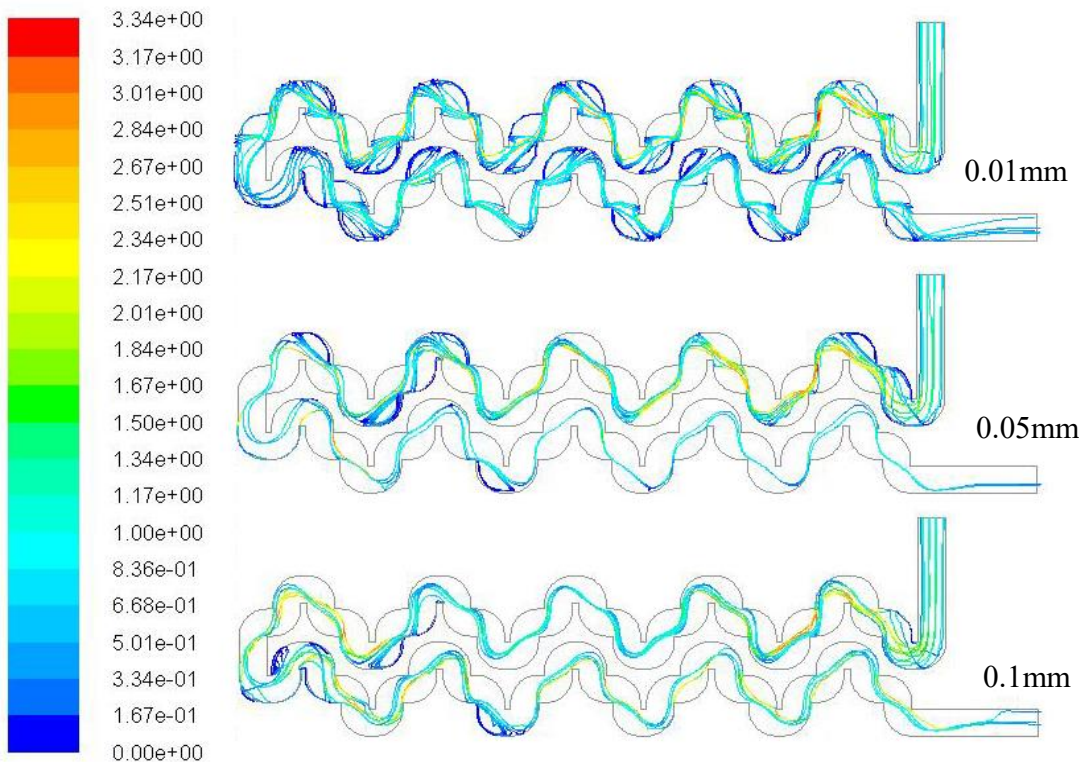

(b)After optimization within emitter nine particle trajectories 
Fig. 4. Emitter flow channel 9 of particle trajectories

of disorder. Some of the particles collide with the wall resulting in reduced particle velocity decrease follow. Some of the particles flow into the vortex area, scroll in vivo cycle of periodic motion. With the emitter clogging chemical substance formed is bound to make the flow path wall roughness increases. This will increase the collision and deposition of particles, allowing particles to adhere to the flow channel.
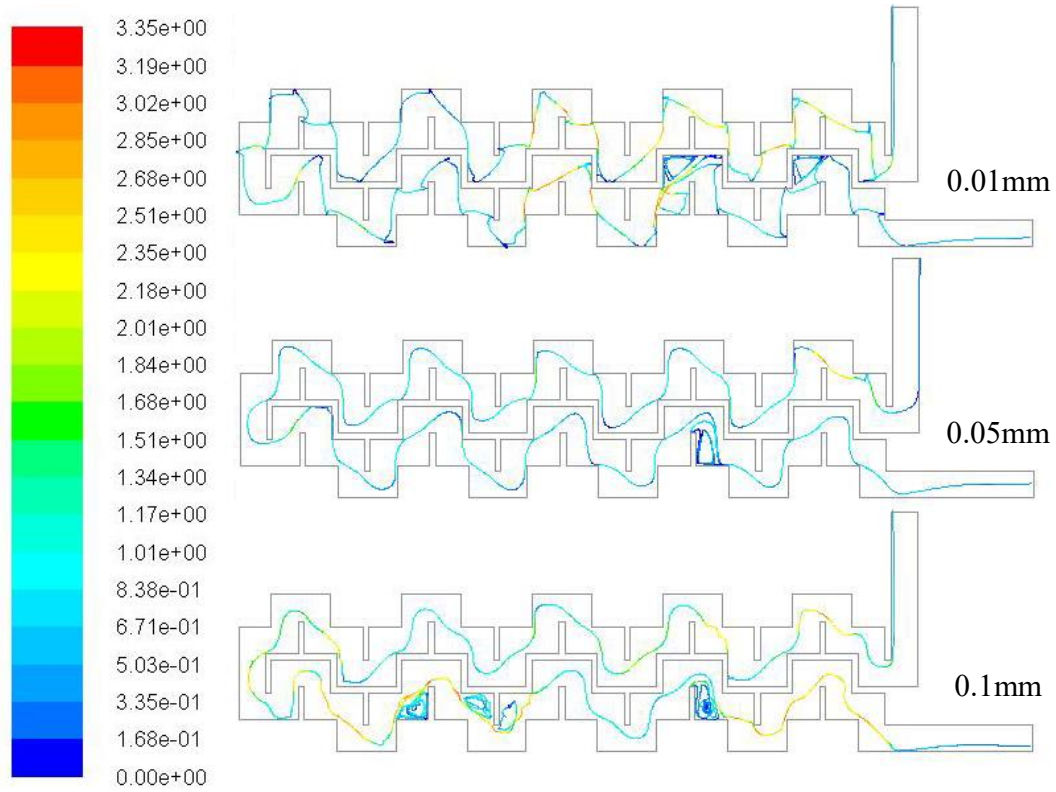

(a) Before optimization within emitter single particle trajectories
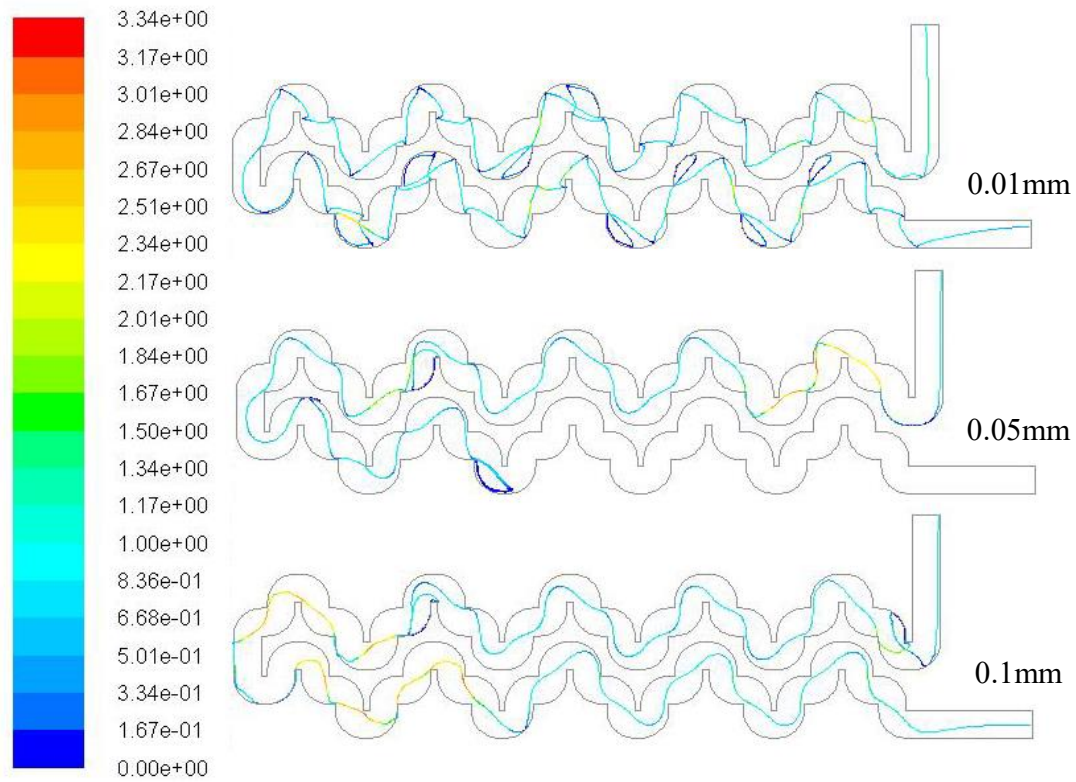

(b)After optimization within emitter single particle trajectories

Fig. 5. Emitter flow velocity vector distribution

Fig. 5 shows the trajectory of particles of different diameters in emitter flow channel. When the particle diameter is $0.01 \mathrm{~mm}$, the particles follow very good, substantially fluid mainstream area coincide and collide with the wall less likely to occur. Due to the small particle diameter it is better to follow, when the particle enters the vortex region after the particles will follow the fluid vortex scroll 
continuously cycle. And gradually decreasing particle velocity close to the speed of the center of the lower scroll area, lost energy from the scroll, the final precipitation occurred in the central region of the scrolls. It is not difficult to infer that over time the body will scroll to the first occurrence of substance clogging the surrounding development. When the particle diameter is increased to $0.05 \mathrm{~mm}$, reduced follow the trajectory of particles in the flow path is beginning to show health disorders, the particles with the wall of the colliding particles become a high probability event. The residence time of particles in the flow channel is reduced, and the probability of particle deposition is reduced. When the particle diameter is further increased to $0.1 \mathrm{~mm}$, the particles in each corner were thrown mainstream flow zones, collisions will occur with the wall at each turning point particles.

\subsubsection{Particles pass rate}

Emitter were DPM model the movement of particles in the flow path, using the discrete phase model to simulate the particles pass rate (Outflow emitter particle number as a percentage of the total number of particles entering).

Figure 2 shows the outflow ratio of two different particles. Data analysis at $10 \mathrm{~m}$ head pressure, when the particle diameter at $0.1 \mathrm{~mm}$, before and after optimization by particle emitters rates of $83.61 \%$, $95.56 \%$ respectively. Because follow particles smaller particles after the collision velocity loss mainstream flow area to gain speed again hit the wall again and so forth. The smaller follow, is not easy to catch and scroll, the probability of staying in the vortex body is smaller, by a higher rate of particles. After optimizing the emitter, the passing rate of particles increased from $81.67 \%$ to 90.28\%.In this case, it's better to follow the particles, it can be a good follow the mainstream area of water flowing out of the emitter. When the particle diameter is $0.05 \mathrm{~mm}$, the pre-optimized and optimized by particle emitter was significantly lower, respectively, 53.61\%, 15.83\%. From above, we can know that when particle diameter between $0.01 \mathrm{~mm}$ to $0.1 \mathrm{~mm}$, the particles neither good track ability, and the department of collision with the ground caused by the occurrence of energy loss, resulting in a portion of the particles retained in the swirl body, continue to lower the rate eventually deposited in the vortex body. Another part of the wall surface of the particles and the occurrence of multiple collisions energy loss is finally continually wall adsorption. Therefore, we can conclude that the particle between $0.01 \mathrm{~mm}$ to $0.1 \mathrm{~mm}$ is the main factor causing clogging.

Table 2. Pass rate of particles under different conditions

\begin{tabular}{lllll}
\hline pressure & Particle diameter & Before optimization & After optimization & Rate increase \\
\hline \multirow{2}{*}{$5 \mathrm{~m}$} & $0.01 \mathrm{~mm}$ & $88.61 \%$ & $91.11 \%$ & $2.50 \%$ \\
& $0.05 \mathrm{~mm}$ & $46.94 \%$ & $81.39 \%$ & $34.44 \%$ \\
& $0.1 \mathrm{~mm}$ & $66.94 \%$ & $95.00 \%$ & $28.06 \%$ \\
\hline \multirow{2}{*}{$10 \mathrm{~m}$} & $0.01 \mathrm{~mm}$ & $81.67 \%$ & $90.28 \%$ & $8.61 \%$ \\
& $0.05 \mathrm{~mm}$ & $53.61 \%$ & $15.83 \%$ & $-37.78 \%$ \\
& $0.1 \mathrm{~mm}$ & $83.61 \%$ & $95.56 \%$ & $11.94 \%$ \\
\hline \multirow{2}{*}{$15 \mathrm{~m}$} & $0.01 \mathrm{~mm}$ & $83.61 \%$ & $86.94 \%$ & $3.33 \%$ \\
& $0.05 \mathrm{~mm}$ & $55.56 \%$ & $3.33 \%$ & $-52.22 \%$ \\
& $0.1 \mathrm{~mm}$ & $83.03 \%$ & $88.33 \%$ & $5.28 \%$ \\
\hline
\end{tabular}

When the particle diameter is $0.05 \mathrm{~mm}$, under different pressure conditions two emitter particles pass rate analysis. When the pressure is $5 \mathrm{~m}$ head, the passing rate increased $34.44 \%$ than before optimized. Vortex flow path area is small enough to maintain the flow rate of the particles continue to stay in the 
vortex zone, so when there is less pressure in the high pass rate of emitter particles. When the head pressure to increase when the $10 \mathrm{~m}$ and $15 \mathrm{~m}$, Vortex flow path area fully developed flow speed, and at this time particles follow and inertia are relatively small, the probability of remaining in the emitter increases. Through internal emitter flow channel water flow field analysis to know within the optimized irrigation flow passage vortex area significantly reduced. The case of imports increased pressure, optimized internal emitter flow velocity vortex region of the wall have a good flushing action. Therefore, that although after optimization by particle emitter was significantly lower, but the scouring effect of the wall was also significantly increased its anti-blocking property needs further study.

\section{Conclusions}

(1) Fractal channel has a better runner complexity of the flow path to achieve full turbulent regime. Emitter flow exponent were 0.487 and 0.489 , two emitter flow regime index is less than 0.5 , and has a good flow regime. Optimized emitter flow regime index change is small, it is considered good emitter hydraulic performance optimized.

(2) After optimization of the emitter area of the flow rate of the mainstream has improved, the velocity of the edge flow rate of $(0.005 \mathrm{~m} / \mathrm{s}-0.752 \mathrm{~m} / \mathrm{s})$, the vortex zone velocity is about $(0.311 \mathrm{~m} / \mathrm{s}-0.930 \mathrm{~m} / \mathrm{s})$ after optimization of the emitter zone of turbulence velocity improved significantly improves the self-cleaning ability emitter.

(3) Practical optimized through rate significantly improved compared to the previous optimization, when the particle diameter is of $0.01 \mathrm{~mm}$ and $0.1 \mathrm{~mm}$. But when the particle diameter is $0.05 \mathrm{~mm}$, it appears opposite phenomenon. Therefore, by reducing the particle emitter rate at $10 \mathrm{~m}$ and $15 \mathrm{~m}$ head also we need to study practical experiments to verify the hypothesis.

\section{Acknowledgment}

Funds for this research was provided by the Water Drip Irrigation and Efficient Water-saving Irrigation Area of Key Technology Research and Demonstration ([2014] 117).

\section{References}

1. Localized irrigation-design, installation, operation, evaluation [M]. Rome: Food and AgricultureOrganization of the United Nations (1980)

2. de Kreij C,van der Burg A M M , Runia W T, Drip irrigation emitter clogging in dutch greenhousesas affected by methane and organic acids [J], Agricultural Water Management,60(2):73 - 85 (2003)

3. Wang Shangjin, Liu Xiaomin, Xi Guang, et al. Agricultural irrigation analysis in Labyrinth Emitter Flow Characteristics [J]. Transactions of the Chinese Society of Agricualtural Engineering, （2000）

4. Luo Jinyao. Theory and technology for water-saving irrigation [M]. Wuhan:Wuhan University Press, (2003)

5. Zhao Wanghua. The key technology of drip irrigation system [J]. Review of China Agricultural Science and Technology, 9 (1):21-25 (2007)

6. Qian Yunbi, Li Yingneng, Yang Gang, et al. Saving Agriculture New Technology [M]. Yellow River Conservancy Press, 12: 8-108(2002) 
7. Jin Hongzhi, Li Guangyong. Abroad overseas trends water-saving irrigation technology and equipment United States 24th International Irrigation Show impressions [J],46-48(2004)

8. Xu Ping, Status and prospects of micro-irrigation technology and equipment market analysis [J]. (1) : 33-36(2002)

9. Wei Zhengying, Zhao Wanhua, Tang Yiping, et al. Anti-clogging design method for the labyrinth channels of drip irrigation emitters [J]. Transactions of the Chinese Society of Agricualtural Engineering,21 (6) : 1-7(2005)

10. Wei Zhengying, Tang Yiping, Wen Juying, et al.Two-phase flow analysis ans experimental inverstigation of micro-PIV and anti-clogging for micro-channels of emitter [J]. Transactions of the Chinese Society of Agricualtural Engineering (Transactions of the CSAE),24（6）: 1-9(2008)

11. Li Yunkai, Yang Peiling, Ren Shumei, et al. Cylindrical Emitter labyrinth flow channel internal fluid flow analysis and numerical simulation [J]. Hydrodynamics Research and Development, Series A, 20 (6) : 736-743(2005)

12. Yan Dazhuang Yang Peiling Ren Shumei Study on dynamic analysis of particle movement in drip emitter based on CFD [J].Transactions of the Chinese Society For Agricultural Machinery, 38 (6):71-74(2007)

13. Zhang Jun. Evaluation of Hydraulic and Anti-clogging Performance and Structural Optimization of Labyrinth-Channel Emitters [D].Xi'an:Xi'an Jiaotong University(2009)

14. Li Yunkai. Experimental research and numerical simulation of flow channel-shaped design and flow characteristics of the emitter points [D]. Beijing:College of Water Resource and Civil Engineering, China Agricualtural Univerity(2005)

15. Glaad Y G, Klous L Z.Hydraulic and Mechanical Properties of Drippers of Drippers[C].Proceedings of the and International Drip Irrigation Congress.July 7-14(1998)

16. Han Quanlong, Zhao Wanghua, Ding Yucheng. Situation with drip Emitter and Analysis [J]. (1) : 17-18(2003)

17. Wei Zhengying.Structural design and rapid develop [ment of labyrinth drip irrigation emitters [D].Xi'an:Xi'an Jiaotong University(2003) 